\title{
La relación escuela - familia y las competencias del docente
}

\section{Escuela familia y competencias docentes}

Leonor Alexandra Rodríguez Álava Mg. Sc.

Universidad Técnica de Manabí, Ecuador

\section{Contacto: alexanroa32@ hotmail.com}

Receptado: 18/07/2013Ａceptado: 26/08/2013

\section{Resumen}

Este artículo presenta un análisis de la relación existente entre las dos instituciones de mayor influencia en la formación de los adolescentes: la familia y la escuela; en las que existe un vínculo inevitable e inexcusable, que se fortalece con el trabajo coordinado y complementario. Los padres confían en la escuela a través del desempeño de los maestros, y los docentes esperan ser aliados de los padres para obtener los mejores resultados; sin embargo, en la mayoría impera la frustración y el desaliento, debilitándose la deseada cooperación. Este artículo tiene como finalidad analizar la necesidad y los fundamentos epistemológicos de la relación escuela - familia y las competencias que el docente debe poseer para fortalecerla; el aporte se direcciona en orientaciones prácticas para la construcción de buenas relaciones desde el desarrollo y formación de la competencia relacionada con la orientación familiar, que favorezca la formación integral de los estudiantes.

Palabras claves: Cooperación familia - escuela, docentes orientadores, educación familiar, rol educativo, relación familia - escuela.

\section{The relationship school - family and the teacher competence}

\begin{abstract}
This article deals with the analysis of the relationship existing between both the family and the school, which are considered the two most influential institutions in the formation of adolescents. There is an unavoidable link-up within the school and the family that is strengthened through the coordinated and complementary work. Parents trust in the school in the same way that teachers hope to be supported by the role of the parents in order to improve
\end{abstract}


Rev. SINAPSIS, Vol. 3, No 2, Diciembre 2013

ISSN $1390-7832$

the work; however in most of them, there comes a feeling of frustration and discouragement, weakening the desired cooperation. This work aims at making an analysis of the need, the epistemological bases of the school-family ration and the skills that teachers should have to firm up such a relation; the contribution is addressed by means of practical guidelines for building up good links from the development and formation of the proficiency related to the family orientation, stimulating the integral formation of the students.

Keywords: family cooperation - school - home teachers - family education - educative role - school family relationship.

\section{Introducción}

A través de la historia, se reconoce a la familia y a la escuela como los dos sistemas más influyentes en el desarrollo personal del individuo; ambas son sistemas insertos en un contexto social. Ríos González, (2009); ambas instituciones también deben dar respuesta a las necesidades que produce una sociedad y promover un efectivo acercamiento que permita incidir sobre los niños y jóvenes.

En concordancia con esto, Cataldo, (1991:17) manifiesta que "La formación de los padres forma parte de la educación de los niños y es un método para promover su desarrollo", por lo que, para que esta situación cambie, las familias tendrán que asumir que la educación es una labor de todos; los docentes deben asumir un papel importante en la atención y capacitación de las familias para mejorar el aprendizaje de los hijos; los cales deben desarrollarse como agentes externos de cambio no encargados de aconsejar o proporcionar información; sino, más bien, responsables de transmitir valores, estrategias y técnicas para que los propios miembros resuelvan sus problemas a través de un proceso de desarrollo personal. Lippitt (1959)

En encuestas realizadas, que se analizan durante el desarrollo de este artículo, se puede corroborar que tanto la competencia de los docentes, como la relación familia - escuela, a pesar de ser adecuada, no es óptima, y que en muchos casos, los docentes no juegan el rol idóneo, lo que evidencia la necesidad de perfeccionar esta importante interacción. Partiendo de este criterio, puede entonces formularse, como problema la insuficiencia en la competencia "orientar a la familia", por parte de los docentes y su incidencia negativa en la relación armónica familia - escuela. 
Rev. SINAPSIS, Vol. 3, No 2, Diciembre 2013

ISSN $1390-7832$

La escuela, por su parte, suele ser el entorno idóneo para ofrecer a las familias información y formación sobre el desarrollo de los hijos. En consonancia y en sentido complementario con lo anteriormente mencionado se plantea como objetivo:

Analizar la necesidad y fundamentos epistemológicos de la relación escuela - familia, asì como la competencia que el docente debe poseer para fortalecer dicha relación.

\section{Desarrollo}

En los actuales escenarios educativos, un profesor no limita su esfera de actuación sólo a exponer un tema en clase; las necesidades sociales, el avance del conocimiento y la innovación en los modelos pedagógicos están exigiendo al ejercicio de más de un papel en la docencia: facilitador, tutor, asesor y orientador para padres y colegas principiantes, entre otros, todo con el objetivo de conseguir la formación integral de sus estudiantes.

En múltiples ocasiones, los mismos docentes comprueban que por falta de adecuada formación, tiempo, o ambas; no pueden ocuparse eficientemente del nuevo rol que le toca asumir, porque difieren por completo de las tareas de enseñanza tradicionalmente asignadas a la profesión. Este profesional requiere de una nueva cultura para lograr excelencia en su desempeño, una cultura que promueva su actualización conforme al contexto social, porque “el desempeño del docente debe llevarse a cabo con orden y organización, estar preparado para enfrentar y resolver imprevistos, anteponer el comportamiento amable, reflejar la buena educación en todos sus actos y lograr con el ejemplo cambio de comportamiento, (Medina Elizondio, 2012)

Tal como se ha mencionado, la formación del docente es un proceso integral, paulatino, gradual y complejo, donde no constituye una excepción el desarrollo de saberes relacionados con la competencia orientar a la familia. Para efectos del presente estudio en la relación escuela - familia, resulta necesario analizar los tipos de competencias que deben poseer los docentes, sobre todo los que trabajan con niños y jóvenes; asi, Perrenoud (2005) selecciona un conjunto de competencias, consideradas como el resultado de una "construcción teórica conectada a la problemática del cambio" que invitan al docente a redescubrirse e inventar, de acuerdo a la realidad de su entorno, experiencia y práctica, y destaca la competencia de informar e implicar a los padres; manifestada en tareas concretas: favorecer reuniones 
Rev. SINAPSIS, Vol. 3, No 2, Diciembre 2013

ISSN $1390-7832$

informativas y de debate, dirigir las reuniones e implicar a los padres en la valorización de la construcción de conocimientos.

Por su parte, Marchesi, A (2007) explica que en la figura docente se intersectan tres esferas y dentro de ellas, se considera la competencia de cooperar con la familia.

Sin embargo; hasta los actuales momentos, de acuerdo a la literatura consultada, no ha sido lo suficientemente tratada la competencia antes mencionada, considerándose ésta como una herramienta de mucha importancia para el logro de los objetivos en la educación actual, o lo que es lo mismo, la formación integral y armónica de los estudiantes. Desde esta perspectiva, el docente como orientador familiar es un profesional de la educación que posee una vocación especial para intervenir en función del bienestar de las familias de sus alumnos, con el mayor respeto y delicadeza. El compromiso con su vocación debe expresarse en el interés por lograr una serie de conocimientos, cualidades, habilidades y destrezas, que lo hagan competente en esta actividad, basando su accionar en principios y valores que persigan como meta la formación integral de sus estudiantes y de las respectivas familias.

Al profundizar en esta problemática, resulta imperante resaltar el papel fundamental que juegan los padres y maestros en la calidad de la educación, partiendo de que la relación familia - escuela es en mucho una materia de actitudes. Los docentes juegan un papel muy importante en el desarrollo de una buena relación (Chispeels, 1996).

El profesorado tiene el poder institucional y ciertos padres pueden sentirse inferiores a ellos, pensando incluso que su intervención puede llegar a perjudicar a los hijos. Los padres rara vez experimentan apoyo social por parte de la escuela, aunque el profesorado diga que lo hace. Fomentar práctica desde los profesores hacia los padres, para darles autoconfianza es por lo tanto muy importante. La comunicación basada en el apoyo social, el elogio, y el ánimo, es crucial; la retroalimentación hacia los padres, debería ser acertada y precisa para evitar malos entendimientos (Nordahl et al 2005). Los padres que sienten que juegan un papel importante ayudando a sus hijos e hijas en el rendimiento académico, y se implican a sí mismos en su educación, pueden también facilitar cambios en los comportamientos de los propios padres y madres (Alter, 1982) 


\section{Competencia orientar a la familia en los docentes}

La competencia orientar a la familia en los docentes, se define como proceso interdisciplinario y transdisciplinario sustentado en los principios de intervención preventiva y desarrollo, encaminado a que la familia como agente educativo asuma la función que le corresponde con la finalidad de facilitar y promover el desarrollo integral de sus hijos para que se constituyan en seres transformadores de sí mismos y de su entorno, en coordinación con el resto de los agentes educativos que intervienen en este proceso.

Uno de los aportes teóricos de esta investigación lo constituyen los subsistemas y componentes que forman la competencia tratada, los cuales, en opinión de la autora son imprescindibles para el logro de los saberes por parte de los docentes y en consecuencia una óptima relación escuela-familia; los que se detallan a continuación:

1. Subsistema Cultural Psico-pedagógico de orientación Familiar,

1.1. Componente 1: Cultura pedagógica

1.2. Componente 2: Psicología de la Familia

2. Subsistema Pedagógico de Orientación Educativa Familiar

2.1. Componente 1: Autogestión del conocimiento

2.2. Componente 2: Comunicación reflexiva

3. Subsistema Axiológico - actitudinal de orientación educativa familiar

3.1. Componente 1: Actitud emocional asertiva

3.2. Componente 2: Congruencia en la actuación personal y social

El primer Subsistema, denominado también Cultural Psico-pedagógico de orientación Familiar, permite desde el saber de la ciencia psicológica y pedagógica y la caracterización precisa de cada ambiente familiar donde conviven los estudiantes y obtener una cultura que brinde la adecuada orientación en pos de una formación integral de los educandos.

Para lograr un conocimiento óptimo y una madurez pedagógica en la orientación familiar de los estudiantes, es preciso poseer una cultura pedagógica, un saber, al decir de Sergio Tobón, y una percepción psicosocial de las familias, Tobón S (2009) plantea en relación a esto que para ello debe existir una adecuada orientación educativa, siendo capaz de desarrollar un proceso interdisciplinario y transdisciplinario sustentado en los principios de intervención preventiva, desarrollo y atención a la diversidad del estudiante, cuyos agentes educativos asumen la función de ser transformadores de sí mismos y de su entorno. 
Por su parte la Psicología de la Familia es un componente de vital importancia para este subsistema, es el conocimiento psicosocial, la caracterización que el docente debe poseer de cada núcleo familiar donde convive cada uno de los estudiantes; gracias a ello el docente puede orientar consecuentemente a todos los padres y tutores de los estudiantes.

Martínez González (1996) puntualiza que en ocasiones se insiste demasiado en la complejidad del estudio de la familia, sin reparar en que estas dificultades son básicamente comunes a todos los objetos de estudio en las Ciencias Sociales. Sin duda, estos obstáculos conducen a la necesaria interdisciplinariedad, multiprofesionalidad y multimetodología.

El segundo subsistma, obedece al saber hacer, es el accionar, el cual persigue una concreción de la competencia orientadora familiar en docentes del nivel básico superior; este subsistema contiene los componentes Autogestión del conocimiento y Comunicación reflexiva..

Autogestión del Conocimiento: establece todas aquellas estrategias que la institución educativa y específicamente el docente, desarrolla como aplicación práctica de los conocimientos adquiridos y que a través de la experiencia le permitirán autogestionar el conocimiento; es decir actuará en base a la metacognición, al respecto, Rios (1990:44), considera que este término es un constructo complejo con el cual se hace referencia al "conocimiento que tiene un sujeto acerca de las estrategias (cognoscitivas) con las que cuenta para resolver un problema y al control que ejerce sobre dichas estrategias para que la solución sea óptima"

Ante lo expuesto, el docente, conforme a las políticas institucionales, utilizará las herramientas necesarias de acuerdo a las necesidades, en unos casos de manera individual y en otros los aplicará en aquellas actividades organizadas por la institución, tales como: reuniones orientadoras a padres, visitas al hogar, las Escuelas de Educación Familiar, charlas, debates, los despachos individuales, el empleo de las redes sociales, entre otras.

Otro de los componentes de este subsistema es la Comunicación reflexiva, un proceso que sirve de herramienta para la orientación familiar; es el camino hacia una comunicación interpersonal efectiva, además del conocimiento de las perspectivas culturales, sin embargo, éste implica valorar las diferencias culturales e individuales, pues la cultura no refleja totalmente la individualidad de la persona y las familias, sino provee el contexto y existen otros muchos componentes que son determinantes, como son el contexto social, la educación, 
Rev. SINAPSIS, Vol. 3, No 2, Diciembre 2013

ISSN $1390-7832$

la clase social, la región, la religión, y las experiencias personales. Todos estos factores son los que llevan al individuo a ver el mundo y comunicarse de manera diferente.

Para llegar a conocer asertiva y efectivamente tanto a las familias como a los estudiantes, se necesita fomentar diálogos que permitan debatir de lo que constituyen prioridades formativas en los alumnos, compartir sus percepciones de la realidad y el significado y los sentimientos asociados a éstas.

El Subsistema axiológico - actitudinal de orientación educativa familiar tiene el objetivo de alcanzar el saber ser como parte de la competencia docente. Se basa fundamentalmente en los valores analizados de manera integral unidos a otros aspectos que rigen el accionar de los seres humanos como son los principios, y las normas establecidas para el logro de una convivencia armónica: "Los valores, junto a los principios y las normas, forman parte de la ética profesional, siendo las formas fundamentales en que la moral presenta las contradicciones, retos o desafíos de la época a dichos profesionales según la naturaleza de su función social (Mendoza, Martìnez, \& Rodrìguez, 2009)

Los seres humanos viven en sociedad, por lo tanto, se requiere de habilidades que les permitan interactuar de manera efectiva, más aún si su campo de actuación profesional es la interacción con otras personas "Las habilidades sociales o competencias sociales, facilitan un control efectivo de las relaciones interpersonales haciendo más hábiles en entender el sentido de los actos de los demás y en saber influir con nuestra conducta en ellos" (Melero, 2000); es decir esta capacidad permite desarrollar valores agregados como la empatía, la cual, según este autor puede describirse como la sensibilidad hacia los sentimientos y preocupaciones de los demás, como la capacidad de "ponerse en su lugar" o de entender su punto de vista. Permite apreciar cuán diferente puede ser la forma de sentir de las personas; aspectos de fundamental importancia para la formación y desarrollo de la competencia orientar a la familia en los docentes.

Este subsistema contiene los componentes actitud emocional asertiva y la congruencia en la actuación personal y social.

Actitud emocional asertiva este componente se enfoca en la actitud que el docente debe tener en su gestión orientadora, consigo mismo y hacia los estudiantes, compañeros docentes, directivos, autoridades y de manera especial con los padres de familia; en este sentido se entiende a la actitud como "el estado de disposición psicológica, adquirida y organizada a 
Rev. SINAPSIS, Vol. 3, No 2, Diciembre 2013

ISSN $1390-7832$

través de la propia experiencia que incita al individuo a reaccionar de una manera característica frente a determinadas personas, objetos o situaciones" (Nieto et al. 2002).

Al referirse a actitud emocional, resulta necesario profundizar en cuestiones tales como las cualidades emocionales y sociales, empleando un enfoque más amplio de la inteligencia, que abarque también la capacidad creativa, el entusiasmo, el talento para la organización y la motivación o las actitudes humanitarias. (Melero, 2000).

Por otro lado, al considerar la asertividad como aspecto que complementa esta actitud, se la valora desde la actitud hacia el propio docente y en su relación con los demás, reflexionando que, en la asertividad interactúan dos manifestaciones: la individual y la social, es decir, aquella en que la persona es capaz por sí misma de asegurar con firmeza sus ideas y convicciones y por otra parte, la capacidad de interactuar en armonía con el resto.

La congruencia en la actuación personal y social: Este componente tiene significativa importancia en la competencia docente, considerando que los valores constituyen un componente de la enseñanza que expresa la significación sobre los fenómenos de la vida material y espiritual, su desarrollo sistemático contribuye a que una persona asuma motivaciones, intereses, normas de conducta, orientaciones valorativas, convicciones y cualidades positivas de la personalidad. Su desarrollo está estrechamente relacionado con la asimilación consciente de los conocimientos y habilidades (Pla Lòpez, 2005)

La finalidad consiste en hacer vida los valores que posee, es decir establecer una relación congruente entre el pensar, decir y actuar, tanto a nivel personal como social, ya que la congruencia de valores se refiere al grado en el cual éstos, las creencias y las metas son similares (Jehn et al., 1999)

En la práctica el modo de actuación se revela en el desempeño del docente en diferentes situaciones y contextos. En su formación debe lograrse la suficiente flexibilidad para transformarse en relación con el desarrollo de la ciencia y la sociedad. (Pla Lòpez, 2005)

Este componente complementa los saberes que debe poseer y llevar a la práctica el docente, los mismos que deben darse de manera sistemática e integral, ya que la adquisición de uno fortalece la consecuencia del otro. 


\section{Metodología}

Para determinar algunos parámetros relacionados con la relación escuela - familia tales como: el grado de satisfacción de los padres, el tipo y profundidad de relación existente, los niveles de preparación de los docentes como guías y promotores de este tipo de relación, se realizó una investigación en la Unidad Educativa ITSUP de la Ciudad de Portoviejo, Manabí, Ecuador, durante el período lectivo 2013 -2014, para lo cual, se diseñaron dos encuestas, una para los padres de familia y otra para los docentes del nivel básico superior.

La primera, es decir, la que se creó para los representantes, padres o tutores de los alumnos, perseguía como objetivo determinar los niveles de satisfacción de éstos con respecto a la relación escuela - familia existente.

La segunda encuesta, se propuso determinar el nivel de preparación de los profesores, como promotores y guías en la relación tratada en este artículo.

Las encuestas se aplicaron a la totalidad de los docentes que impartían clases en el nivel seleccionado, y a un $80 \%$ del total de los padres de familias.

\section{Discusión y análisis de los resultados.}

Los resultados más relevantes de la encuesta aplicada a los padres de familia, se aprecian en las tablas 1, 2 y 3, En la tabla 1 se muestran las respuestas obtenidas de la pregunta “ ¿Cómo es la relación escuela - familia en la institución? Y se aprecia que ninguno de los encuestados, calificó esta relación de mala, la mayoría la evaluaron de buena; también es importante mencionar que el segundo grupo en cantidad, la calificó de muy buena, mientras que un porciento no despreciable que la evalúa de regular y una minoría de excelente.

\begin{tabular}{|r|l|r|r|}
\hline $\mathbf{N}^{\mathbf{0}}$ & \multicolumn{1}{|c|}{ Alternativas } & \multicolumn{1}{c|}{ f } & \multicolumn{2}{|c|}{ \% } \\
\hline 1 & Excelente & 8 & 12,5 \\
\hline 2 & Muy buena & 20 & 31,25 \\
\hline
\end{tabular}


Rev. SINAPSIS, Vol. 3, N², Diciembre 2013

\begin{tabular}{|r|l|r|r|}
\hline 3 & Buena & 24 & 37,5 \\
\hline 4 & Regular & 12 & 18,75 \\
\hline 5 & Mala & 0 & 100 \\
\hline & Total & 64 & \\
\hline
\end{tabular}

Tabla 1. Respuestas dadas por los padres a la pregunta “ ¿Cómo es la relación escuela - familia en la institución?

En la tabla 2 se muestran las respuestas obtenidas de la pregunta “ ¿Con qué frecuencia asiste a la institución? Y se aprecia que la mayoría de los encuestados indicó que asiste cuando el plantel los convoca, un considerable porcentaje manifiestó que asiste a todas las actividades que desarrolla el colegio; también es necesario señalar que un tercer grupo en cantidad, expresa asistir de vez en cuando y una minoría asiste rara vez.

\begin{tabular}{|c|l|r|r|}
\hline $\mathbf{N}^{\mathbf{0}}$ & \multicolumn{1}{|c|}{ Alternativas } & f & \% \\
\hline 2 & A todas las actividades & 16 & 25,00 \\
\hline 1 & Cuando hay convocatorias & 30 & 46,88 \\
\hline 3 & De vez en cuando & 14 & 21,87 \\
\hline 4 & Rara vez & 4 & 6,25 \\
\hline & & 48 & 75,00 \\
\hline
\end{tabular}

Tabla 2. Respuestas dadas por los padres a la pregunta ¿Con qué frecuencia asiste a la institución? 
En la tabla 3 se evidencian las respuestas obtenidas a la pregunta ¿Cómo le gustaría que fuera el vínculo de la institución con los padres de familia? la mayoría de los padres manifestó que éste debe ser de mayor comunicación con los profesores, seguidos por la apreciación que debe haber una mayor participación de los docentes; y otro grupo indica que debe ser de mayor confianza; de la misma manera dos grupos con un mismo porcentaje consideran que continúe de la misma manera y que debe ser de mayor participación de los padres.

\begin{tabular}{|c|l|c|c|}
\hline $\mathbf{N}^{\mathbf{0}}$ & \multicolumn{1}{|c|}{ Alternativas } & f & \% \\
\hline 1 & De mayor confianza & 12 & 18,75 \\
\hline 2 & De mayor comunicación con los profesores & 18 & 28,12 \\
\hline 3 & Que continúe de la misma manera & 10 & 15,62 \\
\hline 4 & De mayor participación de los padres & 10 & 15,62 \\
\hline 5 & De mayor participación de los docentes & 14 & 21,87 \\
\hline & Total & 64 & 99,98 \\
\hline & & & \\
\hline
\end{tabular}

Tabla 3. Respuestas dadas por los padres a la pregunta ¿Cómo le gustaría que fuera el vínculo de la institución con los padres de familia?

En la tabla 4 se demuestran las respuestas obtenidas de los docentes a la pregunta ¿Cree que está preparado para realizar una buena orientación educativa a las familias? 
Rev. SINAPSIS, Vol. 3, N² 2, Diciembre 2013

El mayor porcentaje de docentes indicó que posee poco conocimiento, y con una mínima diferencia la respuesta de nada; apenas una mínima cantidad manifestó estar muy preparado.

\begin{tabular}{|c|c|c|c|}
\hline $\mathbf{N}^{\mathbf{0}}$ & Alternativas & $\mathbf{F}$ & $\mathbf{\%}$ \\
\hline 1 & Mucho & 1 & 6,25 \\
\hline 2 & poco & 8 & 50,00 \\
\hline 3 & Nada & 7 & 43,75 \\
\hline & Total & 16 & 100,00 \\
\hline
\end{tabular}

Tabla 4. Respuestas dadas por los docentes a la pregunta ¿Cree que está preparado para realizar una buena orientación educativa a las familias?

En la tabla 5 se demuestran las respuestas obtenidas de los docentes a la pregunta ¿Qué debe hacer el docente para desarrollar una buena orientación familiar? El mayor porcentaje de docentes indicó que es necesario Generar comunicación, respeto y empatía entre docentes, estudiantes, tutores y autoridades, con el mismo número de respuesta, en segundo lugar, coinciden las opciones de Interesarse en los problemas que tienen los estudiantes y Motivar al padre de familia para que colabore con la escuela; el conseguir el apoyo de la comunidad educativa y la apreciación que esto le corresponde a los orientadores se ubican como opciones que un pequeño porcentaje de maestros consideran como aspectos importantes para la función orientadora a los padres de familia. 
Rev. SINAPSIS, Vol. 3, No 2, Diciembre 2013

\begin{tabular}{|c|c|c|c|}
\hline $\mathbf{N}^{\mathbf{o}}$ & Alternativas & $\mathbf{F}$ & $\%$ \\
\hline 1 & Interesarse en los problemas que tienen los estudiantes & 3 & 18,75 \\
\hline 2 & $\begin{array}{l}\text { Generar comunicación, respeto y empatía entre docentes, estudiantes, } \\
\text { tutores y autoridades }\end{array}$ & 6 & 37,5 \\
\hline 3 & Conseguir el apoyo de la comunidad educativa & 2 & 12,5 \\
\hline 4 & Motivar al padre de familia para que colabore con la escuela & 3 & 18,75 \\
\hline 5 & Le corresponde a los orientadores & 2 & 12,5 \\
\hline & total & 16 & 100 \\
\hline
\end{tabular}

Tabla 5. Respuestas dadas por los docentes a la pregunta ¿Qué debe hacer el docente para desarrollar una buena orientación familiar?

\section{Conclusiones}

La relación familia - escuela, a pesar de ser aceptable, no es la óptima, y en muchos casos, los docentes no juegan el rol idóneo, lo que evidencia la necesidad de perfeccionar esta importante interacción.

Los docentes no poseen los saberes necesarios para desarrollar la función de orientadores educativos de los padres, incidiendo esto en la relación escuela-familia.

\section{Bibliografía}

1. Cataldo, C.Z. (1991): Aprendiendo a ser padres: conceptos y contenidos para el diseño de programas de formación de padres. Madrid : Visor 
2. Marchesi, A. 2007. Sobre el bienestar de los docentesCompetencias, emociones y valores. España: Alianza Editorial.

3. Medina, E. J y D. Barquero. 2012. 20 Competencias profesionales para la práctica docente. Mèxico: Editorial Trillas,S.A, de C.V.

4. Mendoza, A., Martìnez, O., \& Rodrìguez, X. (2009). Formaciòn de valores èticos y morales en el profesional de la salud. Revista Médica Electrónica, 2.

5. Melero, M. P. (2000). La inteligencia emocional en el currìculo de la formaciòn inicial de los maestros. Reviste Interuniversitaria de Formaciòn del Profesorado $N^{o} 38,144$.

6. Nordal, T y S Manger. 2005. Atferdsproblemer blant barn o gunge. Fabbokfolaget

7. Perrenoud, P,H. 2004. Diez nuevas competencias para enseñar. Barcelona, Graò

8. Pla Lòpez, R. (2005). Modo de actuación del docente desde un enfoque integral y contextualizado. Cuba: Instituto Superior Pedagògico Manuel Ascunce DomenenchCiego de Avila.

9. Ríos González, J.A. 2009. Personalidad, madurez humana y contexto familiar. Madrid: CCS.

10. Tobón, S. 2004. Formación basada en competencias: Pensamiento complejo, diseño curricular y Didàctica. .Bogotá, Colombia: ECOE.

11. Tobón, S.. 2006. Las competencias en la edu

12. Zabalza, M.A. 2007. Simulación práctica de la guía para la planificación didáctica de la docencia universitaria. Documento de trabajo 